\title{
Impact of complement component 3/4/5 single nucleotide polymorphisms on renal transplant recipients with antibody- mediated rejection
}

\author{
Zijie Wang ${ }^{1, *}$, Haiwei Yang ${ }^{1, *}$, Miao Guo ${ }^{2, *}$, Zhijian Han ${ }^{1}$, Jun Tao ${ }^{1}$, Hao Chen ${ }^{1}$, Yuqiu \\ $\mathbf{G e}^{3}$, Ke Wang ${ }^{1}$, Ruoyun Tan ${ }^{1}$, Ji-Fu Wei ${ }^{2}$ and Min Gu ${ }^{1}$ \\ ${ }^{1}$ Department of Urology, Nanjing Medical University First Affiliated Hospital, Nanjing 210029, P.R. China \\ ${ }^{2}$ Research Division of Clinical Pharmacology, Nanjing Medical University First Affiliated Hospital, Nanjing 210029, P.R. China \\ ${ }^{3}$ School of Public Health, Nanjing Medical University, Nanjing 211166, P.R. China \\ *These authors have contributed equally to this work \\ Correspondence to: Min Gu, email: njmuwzj1990@hotmail.com \\ Ji-Fu Wei, email: weijifu@hotmail.com \\ Ruoyun Tan, email: tanruoyun112@vip.sina.com \\ Keywords: kidney transplantation, antibody-mediated rejection, complement, single nucleotide polymorphism, next-generation \\ sequencing \\ Received: August 18, $2017 \quad$ Accepted: September 24, $2017 \quad$ Published: October 10, 2017 \\ Copyright: Wang et al. This is an open-access article distributed under the terms of the Creative Commons Attribution License 3.0 \\ (CC BY 3.0), which permits unrestricted use, distribution, and reproduction in any medium, provided the original author and source \\ are credited.
}

\section{ABSTRACT}

Antibody-mediated rejection (ABMR) is an important risk of allograft dysfunction in kidney transplantation. The complement system is considered to be associated with the generation of alloreative antibodies and donor-specific antibodies. However, the association of complement single nucleotide polymorphisms (SNPs) with ABMR still remained unclear. Blood samples of 199 renal transplant recipients containing 68 with $A B M R$ and 131 with stable graft function were collected, and analyzed by next-generation sequencing with an established gene panel. High quality readout was obtained in 18 C3 SNPs, 9 C4 SNPs and 22 C5 SNPs. Concerning C3 gene polymorphisms, after being adjusted with age, sex and immunosuppressive protocols, rs10411506 and rs2230205 were found to be statistically associated with ABMR in dominant model (rs10411506: OR=2.73, 95\% CIs: 1.16, 6.68, $P=0.028$; rs2230205: OR=2.52, 95\% CIs: 1.07, 5.92, $P=0.034$ ); rs10411506, rs2230205 and rs2230201 were found different in HET model (rs10411506: OR=3.05, 95\% CIs: 1.22, 7.64, $P=0.017$; rs2230205: $O R=2.90,95 \%$ CIs: $1.20,7.00, P=0.018$; rs2230201: $O R=2.41$, 95\% CIs: $1.03,5.64, P=0.042$ ). The linkage analysis showed relatively high linkage disequilibrium among these SNPs. In addition, no significant correlation was found between C4 SNPs, or C5 SNPs, and the development of ABMR. Our study firstly identified the two SNPs (rs10411506 and rs2230205) in C3 gene were statistically correlated with ABMR in kidney transplantation. These findings may have implications for the diagnosis and prevention of ABMR.

\section{INTRODUCTION}

Kidney transplantation is the optimal therapy for patients with end-stage renal disease [1]. Despite the advancement in novel immunosuppressive agents and surgical techniques, challenges still remain in the area of maintaining long-term stable allograft function and minimizing the rejection [2]. Among these, the prevention and treatment of antibody-mediated rejection (ABMR) plays a critical role, which has been emerged as an important cause of both short-term and long-term injury to transplanted kidney [3-5]. ABMR often occurs in the 
presence of alloreactive antibodies or donor-specific antibodies (DSAs) and leads to the deterioration in graft function [6]. Efficient measures, such as timely monitoring of alloreative antibodies, maintaining of adequate immunosuppressive agents, have been already taken. However, even with strict adherence, the development of ABMR still persists due to the lack of knowledge in its detailed mechanisms and the sufficiently noninvasive monitoring system for renal transplant recipients $[7,8]$.

ABMR, also known as humoral rejection, is an important cause of short-term and long-term graft injury. The latest Banff criteria for the diagnosis of ABMR include the following three components: detectable DSA, presence of $C 4 \mathrm{~d}$ deposition and histological evidence, including vasculitis and glomerulonephritis [9]. In the pathogenesis of ABMR, endothelial tissue is a key target and damage to the graft is primarily attributable to antigen-antibody complex-mediated activation of the classical complement pathway, which triggers multiple downstream processes, such as the promotion of antigen presentation, recruitment of leukocytes and the promotion of inflammatory processes [10]. Moreover, activation of the complement system in solid organ transplantation often occurs in the acute period during the initial ischemia/ reperfusion phase and the subsequent adaptive immune responses, contributing to the development of ABMR [11].

The complement dependent mechanism plays a vital role in the pathogenesis of ABMR [12]. The antigenantibody complex on graft endothelium activates the classical complement pathway, inducing complement dependent cascade $[9,13]$. The complement cascade leads to the formation of membrane attack complex (MAC) which disrupts the integrity of phospholipid bilayer of cells, killing the cells $[9,13]$. Complement independent mechanisms such as antibody-cell-dependent cytotoxicity (ADCC) can also be mediated by antibodies [13]. Since most of the target antigens present on the endothelium, evidence of acute (glomerulitis, peritubular capillaritis) and chronic (transplant glomerulopathy) microcirculation injury can be found in the biopsy [13]. The damage of endothelium can also lead to the formation of microthrobus, degrading the function of allograft further [9]. In the complement system, all the three pathways, including the classical, alternative and lectin pathways, lead to the activation of $C 3$ component by $C 3$ convertases, release of $C 3 \mathrm{~b}$ opsonin, $C 5$ converstion and eventually membrane attack complex $C 5 \mathrm{~b}-9$ formulation, which is the most critical step in the elaboration of the biological effects of the complement system [14-16]. Therefore, modulation of complement-associated reactions may well determine whether initial activation of the complement sequence eventuates in beneficial or detrimental effects for the recipients.

In recent years, some studies have focused on the influence of $C 3$ genetic polymorphisms on outcomes of kidney transplantation, and certain $C 3$ genotypes were identified. Among these, the role of two C3F allotypes, which are called $C 3 \mathrm{~F}$ (fast) and $C 3 \mathrm{~S}$ (slow), in the short-term and long-term allograft outcomes were the most genotypes reported so far. Mutations from glycine to arginine in a functional region (position 80 ) of $C 3 \mathrm{~F}$ allotype could lead to the variant of $C 3 \mathrm{~S}[17,18]$. This mutation is possibly associated with the ability of $C 3$ to interact with monocyte complement receptors [19]. As an indispensable part in classical activation pathway of the complement, the fourth complement component (C4) is important in the pathogenesis of ABMR in allograft [20]. $C 4 d$ is a complement split protein without biological function formed during the $C 4$ activation and its thioester moiety enables $C 4 \mathrm{~d}$ to bind endothelial cells and basement membrane with strong covalent bonding $[13,21]$. Thus the detection of $C 4 \mathrm{~d}$ on biopsy allograft tissues suggests the classical complement activation and the occurrence of ABMR [22]. In addition, complement $5(C 5)$ is a pivotal complement, which initiates the assembly of the membrane attack complex, and mediates chemotaxis of various immune cells [23]. The progression of complement activation from $C 3$ to $C 5$ results in a soluble cleavage product $C 5 \mathrm{a}$, a highly potent chemoattractant and activator of neutrophils and monocytes [24]. Associated with ABMR, C5a downregulates inhibitory $\mathrm{Fc} \gamma \mathrm{R}$ and up-regulates activating Fc $\gamma \mathrm{R}$ by stimulating macrophages [25]. It is significant to realize that the potentially deleterious effects of the proinflammatory terminal complement component on endothelial cells are controlled by a variety of complement modulators, many of which act on the enzymatic components of $C 3$ and $C 5$ convertases [26]. Recently, a systematic assessment of gene polymorphisms in the complement system, including four $C 3$ allotypes (rs7951, rs11569450, rs11569523 and rs11672613), were performed to investigate the association with graft survival, serum creatinine, delayed graft function and acute rejection of kidney transplantation, and no significant outcome was found [27]. Moreover, previous studies demonstrate that certain genetic variants of $C 5$ are a risk factor for several immune related disorders [28, 29]. As a result, the complement system, containing $C 3$, $C 4$ and $C 5$, may play a crucial role in the development of ABMR episodes; on the other hand, the effects of complement-related single nucleotide polymorphisms (SNPs) still remained largely unknown.

Next-generation sequencing (NGS) technology is a powerful and cost-effective tool for large-scale DNA sequencing, which has already changed the way we think about scientific approaches in genetic and evolutionary research [30]. Compared to conventional method, the primary advantage of NGS technology is the inexpensive production of large volumes of sequence data. Currently, NGS has been applied to an increasing number of human diseases, such as tumors, kidney diseases and obesity [31-33]. 
In our study, by the application of NGS technologies and comprehensive literature review of $C 3 / C 4 / C 5$ genetic polymorphism-related studies, we designed to examine the association between reported $C 3 \mathrm{SNPs}$, as well as $C 4$ and $C 5$ SNPs, and the occurrence of ABMR in kidney transplantation in a Chinese population.

\section{RESULTS}

\section{Baseline characteristics of renal transplant recipients}

The clinical characteristics of these 199 recipients are shown in Table 1. The total incidence of ABMR was $34.17 \%$ (68 out of 199 recipients). Between two renal transplant groups, there was no significant association of PRA and HLA mismatch. In addition, no statistical difference was observed in mean age, gender or immunosuppressive protocols. Among patients in ABMR groups, we further collected ABMR-related clinical information, such as $\mathrm{C} 4 \mathrm{~d}$ scoring, histological classifications and the level of serum DSAs, and reported them in Table 1 . We did not observe any significant differences $(P>0.05)$ in age, sex, donor type and immunosuppressive protocol between the stable and ABMR group.

\section{The association of $C 3$ genotypes and $A B M R$}

A total of 18 reported $C 3$ SNPs were identified using the NGS technology. No deviation from HWE was observed for any $C 3$ polymorphism. The genotypic distributions of the determined $C 3$ polymorphisms in both groups are shown in (Supplementary Table 1).

A logistic regression analysis was performed after controlling for age, sex and immunosuppressive protocols as co-variables in all five analytical models (dominant, recessive, additive, HET and $\mathrm{HOM}$ ) to explore the alternative effects of the variants. For dominant model, rs 10411506 and rs2230205 were found to be significantly associated with the occurrence of ABMR [rs10411506 (GG vs. $\mathrm{GA}+\mathrm{AA}): \mathrm{OR}=2.73,95 \%$ CIs: $1.16,6.68$, $P=0.028$; rs 2230205 (CC vs. CT+TT): $\mathrm{OR}=2.52,95 \%$ CIs: $1.07,5.92, P=0.034$; Table 2 ]. Moreover, for HET model, statistically significant difference was observed in rs10411506, rs2230205 and rs2230201 between two groups [rs10411506 (GG vs. GA): $\mathrm{OR}=3.05,95 \% \mathrm{CIs}$ : $1.22,7.64, P=0.017 ;$ rs 2230205 : $\mathrm{OR}=2.90,95 \%$ CIs: 1.20 , 7.00, $P=0.018$; rs2230201 (CC vs. CT): $\mathrm{OR}=2.41,95 \%$ CIs: $1.03,5.64, P=0.042$; Table 2 ]. In addition, there was no statistical difference between other SNPs in $C 3$ gene and the pathogenesis of ABMR (Table 2). Then, the SNPs of rs11569428, rs2230205, rs116528507, rs10411506, rs4807895, rs2230201 were tested for LD analysis, and the results indicate that these significant SNPs were in high LD status (Figure 1).

\section{The association of $C 4 / C 5$ genotypes and $A B M R$}

A total of 9 C4 SNPs and 22 C5 SNPs were identified. All genotype frequencies of stable group followed HWE. The genetic distributions of $C 4 / C 5$ SNPs screened in ABMR and stable subjects are shown in Supplementary Tables 2 and 3.

After adjusting the age, sex and immunosuppressive protocol, results of correlations between $C 4$ or C5 SNPs and the development of ABMR were presented in Tables 3 and 4. Unfortunately, no significant association was found between the occurrence of ABMR and polymorphisms in $C 4$ or $C 5$ by applying various models.

\section{DISCUSSION}

In this study, we investigated the relationships between reported $C 3 \mathrm{SNPs}$, as well as $C 4$ and $C 5 \mathrm{SNPs}$, and the development of ABMR in renal transplant recipients. Our results showed that rs10411506, rs2230205 and rs2230201 located in C3 gene, especially rs10411506 and rs2230205, were statistically associated with an increased risk of post-transplant ABMR following kidney transplantation. This is the first study to explore the presence and role of complement polymorphisms in ABMR after kidney transplantation.

As an important molecular in innate immune system, $C 3$ is the most abundant component of the complement pathways, which has a great impact on the downstream signals and activities [34]. C3 component and its regulators are well recognized as the crucial factors in the susceptibility to immune-related diseases. Furthermore, multiple studies have shown that $C 3$ SNPs is associated with the pathogenesis of various diseases, such as age-related macular degeneration (AMD), ocular Behcet's disease (BD), Vogt-Koyanagi-Harada syndrome $(\mathrm{VKH})$ and chronic hepatitis $\mathrm{C}$ infection [28, $35,36]$. Among these various studies, the potential role of rs10411506 and rs2230205 were only studied in the pathogenesis of AMD in Chinese population, and the results showed no significant association of rs10411506 and rs2230205 with AMD [35]. However, our study showed that these two SNPs appeared to be an important risk of the ABMR in kidney transplantation. Moreover, recipients carrying with rs10411506 GG genotype were less susceptible to the occurrence of ABMR posttransplantation when compared with those with A allele. Similarly, the rs2230205 CC genotype was found to protect the recipients from experiencing ABMR. Besides, we also found that $C 3$ rs2230201 SNP was statistically associated with the development of ABMR in HET model, which was consistent with previous studies conducted in ocular BD and VKH syndrome, chronic hepatitis C infection and systemic lupus erythematosus [28, 36, 37]. Nevertheless, considering to the negative results of additive model, dominant model and recessive model, 
Table 1: Comparison of baseline characteristics between ABMR and stable subjects

\begin{tabular}{|c|c|c|c|}
\hline Characteristics & Stable group & ABMR group & $P$ value \\
\hline Case number & 131 & 68 & NS \\
\hline Age (years, mean \pm SD) & $37.55 \pm 1.24$ & $38.92 \pm 2.01$ & NS \\
\hline Male (\%) & 62.60 & 55.88 & NS \\
\hline Number of HLA mismatches & $3.52 \pm 0.83$ & $3.41 \pm 0.76$ & NS \\
\hline PRA (\%) & 0.00 & 0.00 & - \\
\hline Immunosuppressive protocol & & & NS \\
\hline Pred + MMF + CsA & 63 & 26 & \\
\hline Pred + MMF + TAC & 59 & 34 & \\
\hline Pred + MMF + CsA + SIR & 5 & 6 & \\
\hline Pred + MMF + TAC + SIR & 4 & 2 & \\
\hline \multicolumn{4}{|l|}{ Type of $A B M R^{*}$} \\
\hline Acute ABMR & - & 23 & \\
\hline Chronic active ABMR & - & 45 & \\
\hline \multicolumn{4}{|c|}{ Grade of morphologic tissue injury } \\
\hline Grade I & - & 25 & \\
\hline Grade II & - & 33 & \\
\hline Grade III & - & 10 & \\
\hline \multicolumn{4}{|l|}{ C4d Scroing by IF } \\
\hline C4d1 & - & 5 & \\
\hline $\mathrm{C} 4 \mathrm{~d} 2$ & - & 17 & \\
\hline $\mathrm{C} 4 \mathrm{~d} 3$ & - & 46 & \\
\hline \multicolumn{4}{|c|}{ Criculating DSAs (MFI, mean $\pm S D$ ) } \\
\hline Class I & - & $1322.15 \pm 545.82$ & \\
\hline Class II & - & $1185.22 \pm 650.08$ & \\
\hline
\end{tabular}

ABMR, antibody-mediated rejection; NS, not significant; SD, standard deviation; HLA, human lymphocyte antigen; PRA, panel reactive antibody; Pred, prednisone; MMF, Mycophenolate Mofetil; CsA, Cyclosporin A; TAC, tacrolimus; SIR, sirolimus.

${ }^{*}$ The classification of ABMR are in accordance with Banff 2007 criteria.

the relative relationship of $\mathrm{rs} 2230201 \mathrm{SNP}$ and ABMR in kidney transplantation by HET model was less convincing when compared with rs10411506 and rs2230205. The linkage analysis further identified the high LD among rs10411506, rs2230205 and rs2230201, which failed to perform the reconstruction of allotype analysis. Given that introns are usually several short sequences that regulate the expression of $C 3$, the rs 10411506 GG genotype and rs2230205 CC genotype may have essential impact on the regulation of $C 3$ protein, thus contributing to the relatively lower risk of ABMR in kidney transplantation [38].

In our study, we failed to observe the significant correlation between $C 4 / C 5$ SNPs and post-transplant
ABMR. SNPs in $C 4$ were considered to be responsible for the differences between $C 4 A$ and $C 4 B$ isotypes, Rodgers and Chido antigenic determinants and to be associated with several autoimmune diseases [39]. A genome-wide association study conducted in healthy Chinese found eight SNPs resided in a 2-Mb MHC region on chromosome 6p21.3 region where RCCX module situates related to copy numbers of $C 4$ gene and one SNP (rs2857009) independently affected the concentration of $C 4$ level in serum [40]. Pertaining to correlations between $C 4$ variations and kidney transplantation, recent studies concentrated on gene copy number variations (CNVs) of $C 4$ and long term graft survival and suggested a possibly 
Table 2: Regression analysis for age-, sex- and immunosuppressive protocol-adjusted C3 genetic polymorphisms among recipients with $\mathrm{ABMR}$

\begin{tabular}{|c|c|c|c|c|}
\hline SNPs & Model & OR & 95\% CIs & $P$ value \\
\hline \multicolumn{5}{|c|}{ rs 17030} \\
\hline & Additive & 1.14 & $0.74,1.75$ & 0.57 \\
\hline & Dominant & 1.39 & $0.67,2.89$ & 0.38 \\
\hline & Recessive & 1.02 & $0.51,2.06$ & 0.95 \\
\hline & HET & 1.43 & $0.66,3.10$ & 0.36 \\
\hline & HOM & 1.31 & $0.54,3.16$ & 0.55 \\
\hline \multicolumn{5}{|c|}{$r s 344555$} \\
\hline & Additive & 1.01 & $0.63,1.63$ & 0.96 \\
\hline & Dominant & 1.26 & $0.68,2.33$ & 0.46 \\
\hline & Recessive & 0.50 & $0.15,1.65$ & 0.25 \\
\hline & HET & 1.44 & $0.76,2.72$ & 0.27 \\
\hline & HOM & 0.60 & $0.17,2.06$ & 0.42 \\
\hline \multicolumn{5}{|c|}{ rs2277984 } \\
\hline & Additive & 1.15 & $0.75,1.78$ & 0.52 \\
\hline & Dominant & 1.39 & $0.67,2.89$ & 0.38 \\
\hline & Recessive & 1.06 & $052,2.14$ & 0.87 \\
\hline & HET & 1.41 & $0.65,3.05$ & 0.38 \\
\hline & $\mathrm{HOM}$ & 1.34 & $0.55,3.26$ & 0.52 \\
\hline \multicolumn{5}{|l|}{ rs 7951} \\
\hline & Additive & 0.97 & $0.47,2.02$ & 0.94 \\
\hline & Dominant & 0.93 & $0.42,2.08$ & 0.86 \\
\hline & Recessive & 1.61 & $0.095,27.33$ & 0.74 \\
\hline & HET & 0.90 & $0.39,2.06$ & 0.80 \\
\hline & HOM & 1.58 & $0.092,26.84$ & 0.75 \\
\hline \multicolumn{5}{|c|}{ rs 2241394} \\
\hline & Additive & 1.40 & $0.53,3.68$ & 0.49 \\
\hline & Dominant & 1.40 & $0.53,3.68$ & 0.49 \\
\hline \multicolumn{5}{|c|}{$r s 2241393$} \\
\hline & Additive & 0.96 & $0.34,2.72$ & 0.94 \\
\hline & Dominant & 1.89 & $0.40,8.94$ & 0.42 \\
\hline \multicolumn{5}{|c|}{ rs 7257062} \\
\hline & Additive & 1.03 & $0.42,2.53$ & 0.95 \\
\hline & Dominant & 1.27 & $0.35,4.63$ & 0.72 \\
\hline & Recessive & 0.61 & $0.061,6.13$ & 0.68 \\
\hline & HET & 1.87 & $0.39,9.05$ & 0.44 \\
\hline & HOM & 0.63 & $0.063,6.27$ & 0.69 \\
\hline \multicolumn{5}{|c|}{$r s 11569536$} \\
\hline & Additive & 1.34 & $0.11,16.35$ & 0.82 \\
\hline
\end{tabular}

(Continued) 


\begin{tabular}{|c|c|c|c|c|}
\hline SNPs & Model & OR & $95 \%$ CIs & $P$ value \\
\hline & Dominant & 1.34 & $0.11,16.35$ & 0.82 \\
\hline \multicolumn{5}{|c|}{$r s 3745568$} \\
\hline & Additive & 1.48 & $0.64,3.43$ & 0.36 \\
\hline & Dominant & 1.48 & $0.64,3.43$ & 0.36 \\
\hline \multicolumn{5}{|c|}{$r s 3745567$} \\
\hline & Additive & 1.45 & $0.57,3.67$ & 0.44 \\
\hline & Dominant & 1.45 & $0.57,3.67$ & 0.44 \\
\hline \multicolumn{5}{|c|}{$r s 2287845$} \\
\hline & Additive & 1.68 & $0.88,3.19$ & 0.12 \\
\hline & Dominant & 1.71 & $0.86,3.39$ & 0.13 \\
\hline & Recessive & 2.68 & $0.15,48.48$ & 0.50 \\
\hline & HET & 1.67 & $0.83,3.35$ & 0.15 \\
\hline & $\mathrm{HOM}$ & 3.01 & $0.17,54.73$ & 0.46 \\
\hline \multicolumn{5}{|c|}{ rs366510 } \\
\hline & Additive & 1.68 & $0.88,3.19$ & 0.12 \\
\hline & Dominant & 1.71 & $0.86,3.39$ & 0.13 \\
\hline & Recessive & 2.68 & $0.15,48.48$ & 0.50 \\
\hline & HET & 1.67 & $0.83,3.35$ & 0.15 \\
\hline & HOM & 3.01 & $0.17,54.73$ & 0.46 \\
\hline \multicolumn{5}{|c|}{$r s 408290$} \\
\hline & Additive & 1.18 & $0.80,3.23$ & 0.40 \\
\hline & Dominant & 1.68 & $0.88,3.23$ & 0.12 \\
\hline & Recessive & 0.93 & $0.42,2.07$ & 0.86 \\
\hline & HET & 2.91 & $1.19,7.16$ & 0.020 \\
\hline & HOM & 1.12 & $0.49,2.54$ & 0.79 \\
\hline \multicolumn{5}{|c|}{$r s 2230205$} \\
\hline & Additive & 1.26 & $0.79,2.01$ & 0.34 \\
\hline & Dominant & 2.52 & $1.07,5.92$ & 0.034 \\
\hline & Recessive & 0.078 & $0.36,1.69$ & 0.53 \\
\hline & HET & 2.90 & $1.20,7.00$ & 0.018 \\
\hline & HOM & 1.73 & $0.61,4.88$ & 0.30 \\
\hline \multicolumn{5}{|c|}{ rs2230204 } \\
\hline & Additive & 1.08 & $0.68,1.69$ & 0.75 \\
\hline & Dominant & 1.35 & $0.70,2.61$ & 0.37 \\
\hline & Recessive & 0.76 & $0.31,1.86$ & 0.55 \\
\hline & HET & 1.49 & $0.75,2.97$ & 0.26 \\
\hline & $\mathrm{HOM}$ & 0.97 & $0.36,2.59$ & 0.94 \\
\hline \multicolumn{5}{|c|}{$r s 10411506$} \\
\hline & Additive & 1.32 & $0.82,2.12$ & 0.26 \\
\hline & Dominant & 2.73 & $1.12,6.68$ & 0.028 \\
\hline
\end{tabular}




\begin{tabular}{lcccc}
\hline SNPs & Model & OR & $\mathbf{9 5 \%}$ CIs & P value \\
\hline Recessive & 0.85 & $0.40,1.82$ & 0.68 \\
$\mathbf{r s 2 2 3 0 2 0 1}$ & HET & $\mathbf{3 . 0 5}$ & $\mathbf{1 . 2 2 , 7 . 6 4}$ & $\mathbf{0 . 0 1 7}$ \\
& HOM & 2.02 & $0.70,5.86$ & 0.20 \\
& & & 0.39 \\
& Additive & 1.23 & $0.77,1.97$ & 0.068 \\
Dominant & 2.15 & $0.95,4.90$ & 0.62 \\
$\boldsymbol{r s 2 2 5 0 6 5 6}$ & Recessive & 0.82 & $0.38,1.78$ & $\mathbf{0 . 0 4 2}$ \\
& HET & $\mathbf{2 . 4 1}$ & $\mathbf{1 . 0 3 , 5 . 6 4}$ & 0.38 \\
& HOM & 1.58 & $0.57,4.36$ & 0.34 \\
& & & 0.49 \\
& Additive & 1.31 & $0.75,2.30$ & 0.29 \\
& Dominant & 1.25 & $0.66,2.34$ & 0.64 \\
& Recessive & 2.75 & $0.43,17.65$ & 0.26 \\
\hline
\end{tabular}

SNPs, single nuclear polymorphisms; OR, odds ratio; CIs: confidential intervals.

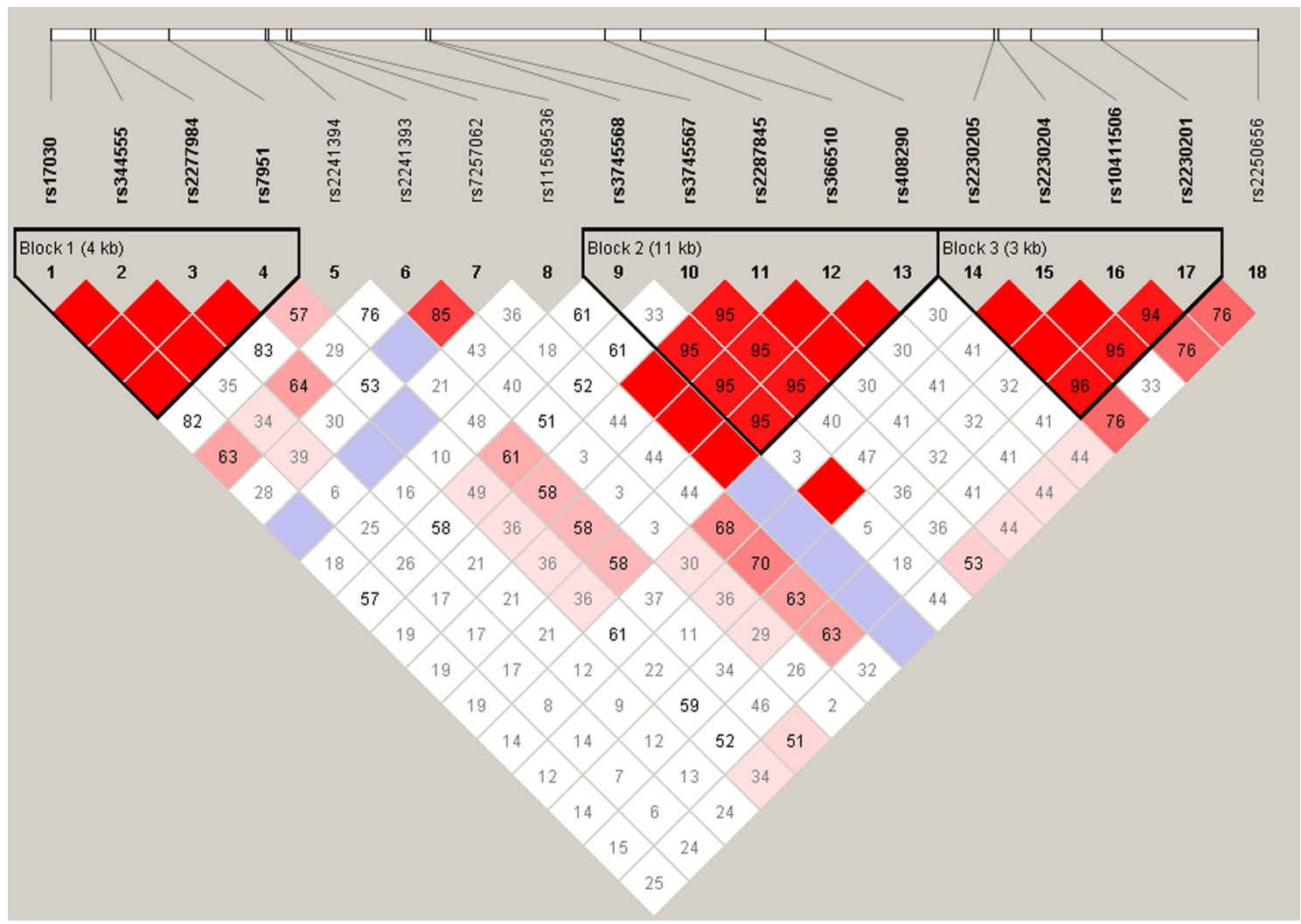

Figure 1: Linkage disequilibrium analysis of rs11569428, rs2230205, rs116528507, rs10411506, rs4807895 and rs2230201 in $C 3$ gene. 
Table 3: Regression analysis of $C 4$ genetic polymorphisms adjusted for age, sex and immunosuppressive protocols in ABMR and stable group

\begin{tabular}{|c|c|c|c|c|}
\hline Position & Model & OR & $95 \%$ CIs & $P$ value \\
\hline \multicolumn{5}{|c|}{ chr6:31963786 } \\
\hline & Additive & 0.73 & $0.24,2.27$ & 0.59 \\
\hline & Dominant & 0.77 & $0.23,2.56$ & 0.67 \\
\hline \multicolumn{5}{|c|}{ chr6:31964228 } \\
\hline & Additive & 1.01 & $0.44,2.36$ & 0.97 \\
\hline & Dominant & 1.17 & $0.46,2.97$ & 0.75 \\
\hline \multicolumn{5}{|c|}{ chr6:31964391 } \\
\hline & Additive & 2.08 & $0.12,36.64$ & 0.62 \\
\hline & Dominant & 2.08 & $0.12,36.64$ & 0.62 \\
\hline \multicolumn{5}{|c|}{ chr6:31964584 } \\
\hline & Additive & 1.49 & $0.62,3.59$ & 0.38 \\
\hline & Dominant & 1.49 & $0.62,3.59$ & 0.38 \\
\hline \multicolumn{5}{|c|}{ chr6:31994974 } \\
\hline & Additive & 1.7 & $0.67,4.31$ & 0.26 \\
\hline & Dominant & 1.7 & $0.67,4.31$ & 0.26 \\
\hline \multicolumn{5}{|c|}{ chr6:31996524 } \\
\hline & Additive & 1.03 & $0.57,1.86$ & 0.94 \\
\hline & Dominant & 1.30 & $0.65,2.59$ & 0.46 \\
\hline \multicolumn{5}{|c|}{ chr6:31996966 } \\
\hline & Additive & 1.07 & $0.58,1.99$ & 0.83 \\
\hline & Dominant & 1.03 & $0.51,2.09$ & 0.93 \\
\hline & Recessive & 1.59 & $0.21,12.02$ & 0.65 \\
\hline & $\mathrm{HOM}$ & 1.59 & $0.21,12.15$ & 0.65 \\
\hline & HET & 1.00 & $0.48,2.05$ & 0.99 \\
\hline \multicolumn{5}{|c|}{ chr6:31997321 } \\
\hline & Additive & 0.78 & $0.14,4.29$ & 0.78 \\
\hline & Dominant & 0.78 & $0.14,4.29$ & 0.78 \\
\hline \multicolumn{5}{|c|}{ chr6:31997401 } \\
\hline & Additive & 0.98 & $0.52,1.82$ & 0.94 \\
\hline & Dominant & 1.01 & $0.53,1.90$ & 0.99 \\
\hline
\end{tabular}

ABMR, antibody-mediated rejection; OR, odds ratio; CIs: confidential intervals.

better prognosis in patients with low dose of $C 4$ gene [41]. The available studies indicate the possibility that C4 SNPs have influence on CNVs of $C 4$ or directly affect the expression of $C 4$, thus contributing to the potential regulation in classical complement pathway and changing the chance of ABMR occurrence.
Recently, JC Jeong et al. [23] carried out the systematic assessment of the complement gene polymorphisms, including seven SNPs in C5 gene (rs12237774, rs2159776, rs17611, rs25681, rs2241004, rs10985126 and rs10818500) and one SNP (rs10404456) in the $C 5 \mathrm{aR}$ gene, on the kidney transplant outcomes, 
Table 4: Regression analysis for age-, sex- and immunosuppressive protocol-adjusted $C 5$ genetic polymorphisms among recipients with $\mathrm{ABMR}$

\begin{tabular}{|c|c|c|c|c|}
\hline SNPs & Model & OR & 95\% CIs & $P$ value \\
\hline \multicolumn{5}{|c|}{ rs76339932 } \\
\hline & Additive & 0.93 & $0.26,3.27$ & 0.90 \\
\hline & Dominant & 0.93 & $0.26,3.27$ & 0.90 \\
\hline \multicolumn{5}{|c|}{ rs12237774 } \\
\hline & Additive & 0.92 & $0.52,1.63$ & 0.78 \\
\hline & Dominant & 0.92 & $0.47,1.79$ & 0.81 \\
\hline & Recessive & 0.83 & $0.14,4.89$ & 0.83 \\
\hline & HET & 0.94 & $0.47,1.87$ & 0.85 \\
\hline & $\mathrm{HOM}$ & 0.81 & $0.13,4.85$ & 0.82 \\
\hline \multicolumn{5}{|c|}{ rs2300931 } \\
\hline & Additive & 1.62 & $0.82,3.15$ & 0.16 \\
\hline & Dominant & 1.59 & $0.74,3.37$ & 0.23 \\
\hline & Recessive & 4.42 & $0.36,53.99$ & 0.24 \\
\hline & HET & 1.44 & $0.65,3.16$ & 0.36 \\
\hline & $\mathrm{HOM}$ & 4.69 & $0.38,57.88$ & 0.23 \\
\hline \multicolumn{5}{|c|}{ rs10985112 } \\
\hline & Additive & 1.03 & $0.29,3.64$ & 0.96 \\
\hline & Dominant & 1.03 & $0.29,3.64$ & 0.96 \\
\hline \multicolumn{5}{|c|}{ rs2269066 } \\
\hline & Additive & 1.05 & $0.60,1.81$ & 0.88 \\
\hline & Dominant & 1.12 & $0.59,2.13$ & 0.73 \\
\hline & Recessive & 0.71 & $0.13,3.94$ & 0.69 \\
\hline & HET & 1.17 & $0.60,2.29$ & 0.64 \\
\hline & HOM & 0.74 & $0.13,4.19$ & 0.74 \\
\hline \multicolumn{5}{|c|}{ rs41260544 } \\
\hline & Additive & 1.44 & $0.38,5.44$ & 0.59 \\
\hline & Dominant & 1.44 & $0.38,5.44$ & 0.59 \\
\hline \multicolumn{5}{|c|}{ rs117287858 } \\
\hline & Additive & 2.13 & $0.29,15.88$ & 0.46 \\
\hline & Dominant & 2.13 & $0.29,15.88$ & 0.46 \\
\hline \multicolumn{5}{|c|}{ rs2230212 } \\
\hline & Additive & 0.75 & $0.31,1.78$ & 0.51 \\
\hline & Dominant & 0.75 & $0.31,1.78$ & 0.51 \\
\hline \multicolumn{5}{|c|}{ rs41311867 } \\
\hline & Additive & 1.44 & $0.38,5.44$ & 0.59 \\
\hline & Dominant & 1.44 & $0.38,5.44$ & 0.59 \\
\hline
\end{tabular}




\begin{tabular}{|c|c|c|c|c|}
\hline SNPs & Model & OR & $95 \%$ CIs & $P$ value \\
\hline \multicolumn{5}{|c|}{ rs 187517049} \\
\hline & Additive & 2.73 & $0.23,31.84$ & 0.42 \\
\hline & Dominant & 2.73 & $0.23,31.84$ & 0.42 \\
\hline \multicolumn{5}{|c|}{ rs12683026 } \\
\hline & Additive & 1.44 & $0.38,5.44$ & 0.59 \\
\hline & Dominant & 1.44 & $0.38,5.44$ & 0.59 \\
\hline \multicolumn{5}{|c|}{ rs10985122 } \\
\hline & Additive & 1.75 & $0.41,7.47$ & 0.45 \\
\hline & Dominant & 1.75 & $0.41,7.47$ & 0.45 \\
\hline \multicolumn{5}{|c|}{ rs41309856 } \\
\hline & Additive & 1.23 & $0.34,4.44$ & 0.75 \\
\hline & Dominant & 1.23 & $0.34,4.44$ & 0.75 \\
\hline \multicolumn{5}{|c|}{ rs144465545 } \\
\hline & Additive & 3.27 & $0.52,20.59$ & 0.21 \\
\hline & Dominant & 3.27 & $0.52,20.59$ & 0.21 \\
\hline \multicolumn{5}{|c|}{ rs41309850 } \\
\hline & Additive & 1.23 & $0.34,4.44$ & 0.75 \\
\hline & Dominant & 1.23 & $0.34,4.44$ & 0.75 \\
\hline \multicolumn{5}{|c|}{ rs181763824 } \\
\hline & Additive & 0.64 & $0.06,6.55$ & 0.71 \\
\hline & Dominant & 0.64 & $0.06,6.55$ & 0.71 \\
\hline \multicolumn{5}{|c|}{ rs2230214 } \\
\hline & Additive & 1.23 & $0.34,4.44$ & 0.75 \\
\hline & Dominant & 1.23 & $0.34,4.44$ & 0.75 \\
\hline \multicolumn{5}{|c|}{ rs10985126 } \\
\hline & Additive & 1.08 & $0.65,1.77$ & 0.77 \\
\hline & Dominant & 1.15 & $0.62,2.15$ & 0.66 \\
\hline & Recessive & 0.90 & $0.25,3.20$ & 0.87 \\
\hline & HET & 1.19 & $0.62,2.30$ & 0.60 \\
\hline & $\mathrm{HOM}$ & 0.96 & $0.26,3.48$ & 0.95 \\
\hline \multicolumn{5}{|c|}{ rs 10985127} \\
\hline & Additive & 1.08 & $0.65,1.77$ & 0.77 \\
\hline & Dominant & 1.15 & $0.62,2.15$ & 0.66 \\
\hline & Recessive & 0.90 & $0.25,3.20$ & 0.87 \\
\hline & HET & 1.19 & $0.62,2.30$ & 0.60 \\
\hline & $\mathrm{HOM}$ & 0.96 & $0.26,3.48$ & 0.95 \\
\hline \multicolumn{5}{|c|}{ rs 28426093} \\
\hline & Additive & 1.85 & $0.82,4.15$ & 0.14 \\
\hline & & & & (Continued) \\
\hline
\end{tabular}




\begin{tabular}{lcccc}
\hline SNPs & Model & OR & $\mathbf{9 5 \%}$ CIs & P value \\
\hline \multirow{2}{*}{ rs10818499 } & Dominant & 1.57 & $0.59,4.18$ & 0.37 \\
& & & \\
& Additive & 1.14 & $0.77,1.70$ & 0.51 \\
Dominant & 1.54 & $0.81,2.92$ & 0.19 \\
& Recessive & 0.89 & $0.42,1.85$ & 0.75 \\
HET & 1.81 & $0.89,3.67$ & 0.10 \\
rs17216529 & 1.19 & $0.52,2.70$ & 0.69 \\
& & & 0.67 \\
& HOM & 0.89 & $0.51,1.54$ & 0.86 \\
& Additive & 0.94 & $0.49,1.81$ & 0.45 \\
Dominant & 0.52 & $0.10,2.78$ & 0.95 \\
Recessive & 1.02 & $0.52,2.03$ & 0.46 \\
\hline
\end{tabular}

SNPs, single nuclear polymorphisms; OR, odds ratio; CIs: confidential intervals.

showing that the GGCG allotype of $C 5$ in both recipients and donors was associated with lower renal allograft, whereas $C 5 \mathrm{aR}$ genotypes of recipients were not associated with acute rejection, and there was also no statistically significant association between donor $C 5 / C 5 \mathrm{aR}$ genotypes and acute rejection function [42]. Importantly, this study focused on the long-term outcomes of renal transplant and acute rejection, instead of the subgroup analysis of ABMR from the acute rejection, which is more correlated with the activation and progress of the complement system. However, being restricted with the collected samples, we could not perform quantitative evaluation of serum $C 5$ in two groups to validate our outcomes in genetic polymorphisms, which requires further prospective research.

Recent study conducted by Ermini [27] focused on the influence of SNPs in complement system in the short-term and long-term outcomes of renal transplant, including delayed graft function, acute rejection, graft survival and serum creatinine, instead of the subgroup analysis of ABMR from the acute rejection, which is more correlated with the activation and progress of the complement system. Nevertheless, the case number of eligible recipients in ABMR and stable groups of our transplant center is limited, leading to the potential bias of our outcomes. Therefore, a large-scale, multi-center and well-designed study of the association of $C 3$ SNPs and ABMR in renal transplant recipients should be conducted in the future.

In summary, we show here for the first time that the rs10411506 and rs2230205 in $C 3$ gene are statistically correlated with the development of ABMR in renal transplant recipients, and no significant relationship of $C 4$ or $C 5$ SNPs were observed during the episodes of posttransplant ABMR. These findings may have implications for the diagnosis and prevention of ABMR, contributing to the promotion of the graft survival and patients' life quality in kidney transplantation.

\section{MATERIALS AND METHODS}

\section{Ethics statement}

The study protocol was in accordance with the ethical standards of the Declarations of Helsinki and Istanbul. Being limited to the living-related transplantation of kidney tissues to their lineal or collateral relative not beyond the third degree of kinship or the cadaveric allograft donors of cardiac death (DCD), the protocol of this study was approved by the local Ethics Committee of the First Affiliated Hospital with Nanjing Medical University, and written informed consent was obtained from all transplant recipients. None of the transplant donors were from a vulnerable population, and all donors or next of kin freely provided written informed consent.

\section{Study design and subjects}

\section{Study design}

This was a 12-month, retrospective, case-control trial containing 199 renal transplant recipients who underwent kidney transplantation between February $1^{\text {st }}$, 2008 and December $1^{\text {st }}, 2015$ in renal transplant center 
of the First Affiliated Hospital with Nanjing Medical University. This study was designed to investigate the distributions of $C 3 / C 4 / C 5$ SNPs between patients with period of ABMR and stable allograft function. The inclusion criteria to select the patients from stable group were as follows: [1]. The follow-up duration was longer than at least six months, and had never experienced the period of acute rejection, delayed graft dysfunction (DGF) or opportunistic infection; [2]. The concentration of serum creatinine (Scr) was lower than $120 \mu \mathrm{mol} / \mathrm{L}(1.36 \mathrm{mg} / \mathrm{dl})$ for at least three months at the time of enrollment; [3]. Patients aged from 18 years old to 60 years old. Patients with following exclusive criteria were excluded in stable group: [1]. Patients aged less than 18 years old or older than 60 years old; [2]. History of acute rejection, DGF or opportunistic infection; [3]. Fluctuation of Scr over than $120 \mu \mathrm{mol} / \mathrm{L}(1.36 \mathrm{mg} / \mathrm{dl})$ during the last three months of enrollment; [4]. Pregnant women and active HIV infection; [5]. Chronic lung disease requiring supplemental oxygen therapy. To enroll patients into the ABMR group, patients with significant clinical characteristics, such as an increase in serum creatinine level by $20 \%$ from baseline (not attributable to other cases) and overloaded urine protein, were required to perform the indication allograft biopsy immediately before the administration of highdose steroids therapy. The diagnostic criteria of ABMR were mainly based on the comprehensive histological examination according to Banff 07 classification [43].

\section{Data collection}

Medical records were critically reviewed and related data, including age, gender, transplant date, duration of transplantation, transplant times, immunosuppressive protocol, were extracted by at least two clinicians for patient selection. Data on panel reactive antibody (PRA) and human leukocyte antigen (HLA) mismatch during pretransplant period were also collected.

\section{Subjects}

Intravenous infusion of $500 \mathrm{mg} / \mathrm{d}$ of methylprednisolone was used during the surgery and up until 2 days after the operation. Then the dosage was reduced to $400 \mathrm{mg}, 300 \mathrm{mg}, 200 \mathrm{mg}$ and $80 \mathrm{mg}$ each subsequent day, followed by prednisone $30 \mathrm{mg} / \mathrm{d}$ as a maintenance therapy. In addition, Basiliximab (20 mg) was intravenously used at 30 minutes before the operation and the fourth day after the operation, respectively. All recipients received a three-drug or four-drug immunosuppressive regimen: Cyclosporin A $(\mathrm{CsA})(\mathrm{n}=100)$ or tacrolimus $(\mathrm{n}=99)$ in combination with mycophenolate mofetil (MMF) and prednisone, with or without sirolimus $(n=17)$. The dosage of CsA and tacrolimus was started at $8 \mathrm{mg} / \mathrm{kg} / \mathrm{d}$ and $0.2 \mathrm{mg} / \mathrm{kg} / \mathrm{d}$, respectively, and then adjusted according to results of therapeutic drug monitoring the serum creatinine levels. A dosage of $200 \mathrm{mg} / \mathrm{d}$ of intravenous methylprednisolone was adopted for ABMR episodes with three to five days.

\section{Sample collection, preparation and NGS}

Peripheral blood samples $(2 \mathrm{ml})$ from each recipient included in our study were collected with $\mathrm{BD}$ Vacutainer tubes containing sodium heparin when they were admitted to our center before the renal biopsy. Then, each collected blood sample was immediately transferred to the laboratory and stored at $-80^{\circ} \mathrm{C}$. The DNA of subjects was extracted from collected peripheral blood samples using QIAmp DNA Mini Kit (Qiagen, Hilden, Germany). Quantitative detection of concentration and purity of genomic DNA (gDNA) was performed by NanoDrop ND2000 (Thermo, MA, USA), while the gene integrity was tested by agarose gel electrophoresis. Requirements for acceptable gDNA were as follows: total mass $\geq 1 \mu \mathrm{g}$, absorbance ratio A260/A280 at $\geq 1.80$ and $\leq$ 2.0. Then a pool containing upstream and downstream oligonucleotides specific to the targeted regions of interest was hybrids to the gDNA samples. Then gDNA was fragmented using a Bioruptor Interrupt instrument (Diagenode, Belgium) and quantitative detection was performed to ensure average fragment size of $150 \mathrm{bp}$ to $250 \mathrm{bp}$. Fragmentation was followed by end repair, dA tailing, and sequencing adaptor ligation by ABI 9700 PCR instrument (ABI, USA). The adapter-ligated DNA was amplified by selective, limited-cycle PCR for 5 cycles and then quantitatively analyzed using Qubit dsDNA HS Assay Kit (Invitrogen, USA). Prepared library (750ng) was hybridized with $11 \mu$ hybridization block (Allwegene, China), 20 $\mu$ l hybridization buffer (Allwegene, China) and a mix of $5 \mu \mathrm{l}$ RNase block (Invitrogen, USA) and $2 \mu$ l Probe (Allwegene, China) for overnight (at least 8-16h) at $65^{\circ} \mathrm{C}$. The hybridized products were mixed with $200 \mu$ l nabeads MyOne Streptavidin T1 magnetic beads (Invitrogen, USA) for $30 \mathrm{~min}$ at room temperature. After two times of washing by wash buffer (Allwegene, China), the mixture was amplified for 16 PCR cycles and quantitatively assessed using Qubit dsDNA HS Assay Kit (Invitrogen, USA). Captured libraries were denatured and loaded onto an Illumina cBot instrument at 12 to $16 \mathrm{pmol} / \mathrm{L}$ for cluster generation according to the manufacturer's instructions. Up to 20 WUCaMP libraries were sequenced per HiSeq lane. A PhiX control (Illumina) was added to lane 8 of each flowcell.

\section{Analysis of NGS data}

Sequencing data, such as the number of altered chromosomes, genomic alternation information and the determination of the depth of sequencing coverage, were analyzed. All analyzed were based on the human reference sequence UCSC build hg19 (NCBI build 37.2) using the Burrows-Wheeler Aligner (BWA) [44]. Local alignment and duplication removal were completed by the application of the Genome Analysis Tool Kit (GATK) and Picard software. Detection of SNPs was performed using dbSNP 132. Damaging or deleterious SNPs were predicted 
using the Gemini software, and prediction tools, including sorting intolerant from tolerant (SIFT) and polymorphism phenotyping (PolyPhen) were used for the analysis of all human non-synonymous SNPs. In addition, putative somatic variant calls were detected with two separate programs, MuTect 1.1.5 and VarScan 2.3.6 softwares, pairing each sample with its matched blood.

\section{Statistical analysis}

Hardy-Weinberg equilibrium (HWE) was analyzed using gene frequencies obtained by a single gene counting. Chi-square test was used to compare observed and expected values. Genotype association analysis was performed using dominant model (minor allele homozygotes plus heterozygotes vs. major allele homozygotes), recessive (minor allele homozygotes vs. heterozygotes plus major homozygotes), additive model (major homozygotes vs. heterozygotes vs. minor homozygotes), HET model (major homozygotes vs. heterozygotes) and HOM model (major homozygotes vs. minor homozygotes). Genotypic frequencies comparisons between control and ABMR groups were assessed by the chi-square test. In addition, we explored linkage disequilibrium (LD) blocks using Haploview version 4.2 software. Odds ratios (OR) and $95 \%$ confidence intervals (95\% CIs) were calculated by SPSS 13.0 software (SPSS Inc., Chicago, IL, USA). $P<0.05$ was considered significant. The OR provides an effect estimate, the value of which less than 1 is considered as a protective effect, whereas the value more than 1 is associated with an increased risk. In addition, the genotypic distributions of the $C 3 \mathrm{SNPs}$ in ABMR recipients and in stable subjects were analyzed with logistic regression models adjusted for age, sex and immunosuppressive protocol.

\section{Abbreviations}

ABMR: antibody-mediated rejection; HLA: human leukocyte antigens; MHC: major histocompatibility complex; DSA: donor-specific antibodies; PRA: panel reactive antibody; SNP: single nucleotide polymorphisms; HWE: Hardy-Weinberg equilibrium; NGS: next-generation sequencing; AMD: age-related macular degeneration; BD: Behcet's disease; VKH: Vogt-Koyanagi-Harada syndrome; CNV: copy number variations; DCD: donors of cardiac death; DGF: delayed graft dysfunction; Scr: serum creatinine; CsA: Cyclosporin A; MMF: mycophenolate mofetil; SIFT: sorting intolerant from tolerant; GATK: Genome Analysis Tool Kit; LD: linkage disequilibrium; OR: odds ratios; 95\%CIs: 95\% confidence intervals.

\section{Author contributions}

Zijie Wang: genetic analysis and manuscript preparation;
Haiwei Yang: genetic analysis and study design; Miao Guo: genetic analysis and data interpretation; Zhijian Han: sample collection;

Jun Tao: sample collection;

Hao Chen: sample collection and data analysis;

Yuqiu Ge: data analysis;

Chunchun Zhao: data interpretation;

Chuanjian Suo: sample collection;

Ke Wang: data analysis;

Shuhui Si: genetic analysis;

Ruoyun Tan: manuscript preparation and foundation collection;

Jifu Wei: study design and foundation collection;

Min Gu: study design, manuscript preparation and foundation collection;

All authors reviewed this manuscript.

\section{FUNDING}

This work was supported by the National Natural Science Foundation of China [grant numbers 81570676 , 81100532, 81470981], the Science and Education Health Project of Jiangsu Province for Important Talent [grant number RC2011055], the "333 High Level Talents Project" in Jiangsu Province, China [grant numbers BRA2015469, BRA2016514 (2011 and 2013)], the Standardized Diagnosis and Treatment Research Program of Key Diseases in Jiangsu Province, China [grant number BE2016791], the Open Project Program of Health Department of Jiangsu Province, China [grant number JSY-2-2016-099], the Jiangsu Province Six Talents Peak from Department of Human Resources, Social Security Office of Jiangsu Province, China [grant numbers 2010WSN-56, 2011-WS-033], the General Program of Health Department of Jiangsu Province, China [grant number H2009907], and the Priority Academic Program Development of Jiangsu Higher Education Institutions [grant number JX10231801]. National Key R\&D Plan for Precision Medicine [grant number 2017YFC0910001].

\section{CONFLICTS OF INTEREST}

The authors have declared that no competing interests exist.

\section{REFERENCES}

1. Wang Z, Han Z, Tao J, Lu P, Liu X, Wang J, Wu B, Huang Z, Yin C, Tan R, Gu M. Clinical efficacy and safety of pamidronate therapy on bone mass density in early postrenal transplant period: a meta-analysis of randomized controlled trials. PLoS One. 2014; 9:e108106.

2. Liu X, Liu K, Wang Z, Liu C, Han Z, Tao J, Lu P, Wang J, Wu B, Huang Z, Yin C, Gu M, Tan R. Advanced glycation end products accelerate arteriosclerosis after renal 
transplantation through the AGE/RAGE/ILK pathway. Exp Mol Pathol. 2015; 99:312-9.

3. Terasaki PI, Cai J. Human leukocyte antigen antibodies and chronic rejection: from association to causation. Transplantation. 2008; 86:377-83.

4. Colvin RB. Antibody-mediated renal allograft rejection: diagnosis and pathogenesis. J Am Soc Nephrol. 2007; 18:1046-56

5. Magil AB, Tinckam K. Monocytes and peritubular capillary C4d deposition in acute renal allograft rejection. Kidney Int. 2003; 63:1888-93.

6. Halloran PF, Famulski KS, Chang J. A probabilistic approach to histologic diagnosis of antibody-mediated rejection in kidney transplant biopsies. Am J Transplant. 2017; 17:129-39.

7. Ng YW, Singh M, Sarwal MM. Antibody-mediated rejection in pediatric kidney transplantation: pathophysiology, diagnosis, and management. Drugs. 2015; 75:455-72.

8. Naesens M, Khatri P, Li L, Sigdel TK, Vitalone MJ, Chen R, Butte AJ, Salvatierra O, Sarwal MM. Progressive histological damage in renal allografts is associated with expression of innate and adaptive immunity genes. Kidney Int. 2011; 80:1364-76.

9. Kim M, Martin ST, Townsend KR, Gabardi S. Antibodymediated rejection in kidney transplantation: a review of pathophysiology, diagnosis, and treatment options. Pharmacotherapy. 2014; 34:733-44.

10. Muduma G, Odeyemi I, Smith-Palmer J, Pollock RF. Review of the clinical and economic burden of antibodymediated rejection in renal transplant recipients. Adv Ther. 2016; 33:345-56.

11. Sacks SH, Zhou W. The role of complement in the early immune response to transplantation. Nat Rev Immunol. 2012; 12:431-42.

12. Colvin RB, Smith RN. Antibody-mediated organ-allograft rejection. Nat Rev Immunol. 2005; 5:807-17.

13. Puttarajappa C, Shapiro R, Tan HP. Antibody-mediated rejection in kidney transplantation: a review. J Transplant. 2012; 2012:193724.

14. Koscielska-Kasprzak K, Bartoszek D, Myszka M, Zabinska $\mathrm{M}$, Klinger $\mathrm{M}$. The complement cascade and renal disease. Arch Immunol Ther Exp (Warsz). 2014; 62:47-57.

15. Walport MJ. Complement. First of two parts. N Engl J Med. 2001; 344:1058-66.

16. Fearon DT, Daha MR, Strom TB, Weiler JM, Carpenter $\mathrm{CB}$, Austen KF. Pathways of complement activation in membranoproliferative glomerulonephritis and allograft rejection. Transplant Proc. 1977; 9:729-39.

17. Teisberg P. High voltage agarose gel electrophoresis in the study of C 3 polymorphism. Vox Sang. 1970; 19:47-56.

18. Alper CA, Johnson AM, Birtch AG, Moore FD. Human C'3: evidence for the liver as the primary site of synthesis. Science. 1969; 163:286-8
19. Arvilommi H. Capacity of complement c3 phenotypes to bind on to mononuclear cells in man. Nature. 1974; 251:740-1.

20. Murata K, Baldwin WM 3rd. Mechanisms of complement activation, C4d deposition, and their contribution to the pathogenesis of antibody-mediated rejection. Transplant Rev (Orlando). 2009; 23:139-50.

21. Nickeleit V, Mihatsch MJ. Kidney transplants, antibodies and rejection: is $\mathrm{C} 4 \mathrm{~d}$ a magic marker? Nephrol Dial Transplant. 2003; 18:2232-9.

22. Collins AB, Schneeberger EE, Pascual MA, Saidman SL, Williams WW, Tolkoff-Rubin N, Cosimi AB, Colvin RB. Complement activation in acute humoral renal allograft rejection: diagnostic significance of C4d deposits in peritubular capillaries. J Am Soc Nephrol. 1999; 10:2208-14.

23. Jeong JC, Hwang YH, Kim H, Ro H, Park HC, Kim YJ, Kim MG, Ha J, Park MH, Chae DW, Ahn C, Yang J. Association of complement 5 genetic polymorphism with renal allograft outcomes in Korea. Nephrol Dial Transplant. $2011 ; 26: 3378-85$.

24. Coulthard LG, Woodruff TM. Is the complement activation product $\mathrm{C} 3 \mathrm{a}$ a proinflammatory molecule? Re-evaluating the evidence and the myth. J Immunol. 2015; 194:3542-8.

25. Konrad S, Engling L, Schmidt RE, Gessner JE. Characterization of the murine IgG Fc receptor III and IIB gene promoters: a single two-nucleotide difference determines their inverse responsiveness to C5a. J Biol Chem. 2007; 282:37906-12.

26. Baldwin WM 3rd, Valujskikh A, Fairchild RL. Mechanisms of antibody-mediated acute and chronic rejection of kidney allografts. Curr Opin Organ Transplant. 2016; 21:7-14.

27. Ermini L, Weale ME, Brown KM, Mesa IR, Howell WM, Vaughan R, Chowdhury P, Sacks SH, Sheerin NS. Systematic assessment of the influence of complement gene polymorphisms on kidney transplant outcome. Immunobiology. 2016; 221:528-34.

28. Xu D, Hou S, Zhang J, Jiang Y, Kijlstra A, Yang P. Copy number variations and gene polymorphisms of complement components in ocular Behcet's disease and Vogt-KoyanagiHarada syndrome. Sci Rep. 2015; 5:12989.

29. Giles JL, Choy E, van den Berg C, Morgan BP, Harris CL. Functional analysis of a complement polymorphism (rs17611) associated with rheumatoid arthritis. J Immunol. 2015; 194:3029-34.

30. Metzker ML. Sequencing technologies - the next generation. Nat Rev Genet. 2010; 11:31-46.

31. Kumondai $\mathrm{M}$, Hosono $\mathrm{H}$, Orikasa $\mathrm{K}$, Arai $\mathrm{Y}$, Arai T, Sugimura H, Ozono S, Sugiyama T, Takayama T, Sasaki T, Hirasawa N, Hiratsuka M. CYP2A13 genetic polymorphisms in relation to the risk of bladder cancer in Japanese smokers. Biol Pharm Bull. 2016; 39:1683-6.

32. Paolini B, Maltese PE, Del Ciondolo I, Tavian D, Missaglia S, Ciuoli C, Zuntini M, Cecchin S, Bertelli M, Pompucci G. 
Prevalence of mutations in LEP, LEPR, and MC4R genes in individuals with severe obesity. Genet Mol Res. 2016.

33. Edrees BM, Athar M, Al-Allaf FA, Taher MM, Khan W, Bouazzaoui A, Al-Harbi N, Safar R, Al-Edressi H, Alansary K, Anazi A, Altayeb N, Ahmed MA, Abduljaleel Z. Next-generation sequencing for molecular diagnosis of autosomal recessive polycystic kidney disease. Gene. 2016; 591:214-26.

34. Mullins RF, Russell SR, Anderson DH, Hageman GS. Drusen associated with aging and age-related macular degeneration contain proteins common to extracellular deposits associated with atherosclerosis, elastosis, amyloidosis, and dense deposit disease. FASEB J. 2000; 14:835-46.

35. Pei XT, Li XX, Bao YZ, Yu WZ, Yan Z, Qi HJ, Qian T, Xiao HX. Association of c3 gene polymorphisms with neovascular age-related macular degeneration in a chinese population. Curr Eye Res. 2009; 34:615-22.

36. Chowdhury SJ, Karra VK, Gumma PK, Bharali R, Kar P. rs2230201 polymorphism may dictate complement C3 levels and response to treatment in chronic hepatitis $\mathrm{C}$ patients. J Viral Hepat. 2015; 22:184-91.

37. Miyagawa H, Yamai M, Sakaguchi D, Kiyohara C, Tsukamoto H, Kimoto Y, Nakamura T, Lee JH, Tsai CY, Chiang BL, Shimoda T, Harada M, Tahira T, et al. Association of polymorphisms in complement component $\mathrm{C} 3$ gene with susceptibility to systemic lupus erythematosus. Rheumatology (Oxford). 2008; 47:158-64.

38. Rodriguez-Trelles F, Tarrio R, Ayala FJ. Origins and evolution of spliceosomal introns. Annu Rev Genet. 2006; 40:47-76.
39. Blanchong CA, Chung EK, Rupert KL, Yang Y, Yang Z, Zhou B, Moulds JM, Yu CY. Genetic, structural and functional diversities of human complement components $\mathrm{C} 4 \mathrm{~A}$ and $\mathrm{C} 4 \mathrm{~B}$ and their mouse homologues, Slp and C4. Int Immunopharmacol. 2001; 1:365-92.

40. Yang X, Sun J, Gao Y, Tan A, Zhang H, Hu Y, Feng J, Qin X, Tao S, Chen Z, Kim ST, Peng T, Liao M, et al. Genomewide association study for serum complement $\mathrm{C} 3$ and $\mathrm{C} 4$ levels in healthy Chinese subjects. PLoS Genet. 2012; 8:e1002916.

41. Bay JT, Schejbel L, Madsen HO, Sorensen SS, Hansen JM, Garred P. Low C4 gene copy numbers are associated with superior graft survival in patients transplanted with a deceased donor kidney. Kidney Int. 2013; 84:562-9.

42. Gunesacar R, Opelz G, Erken E, Dohler B, Ruhenstroth A, Susal C. Complement C5a receptor gene $450 \mathrm{C} / \mathrm{T}$ polymorphism in renal transplant recipients: association of the CT genotype with graft outcome. Tissue Antigens. 2015; 85:104-7.

43. Solez K, Colvin RB, Racusen LC, Haas M, Sis B, Mengel M, Halloran PF, Baldwin W, Banfi G, Collins AB, Cosio F, David DS, Drachenberg C, et al. Banff 07 classification of renal allograft pathology: updates and future directions. Am J Transplant. 2008; 8:753-60.

44. Li H, Durbin R. Fast and accurate long-read alignment with Burrows-Wheeler transform. Bioinformatics. 2010; 26:589-95. 